\title{
Juntos forjamos el futuro del planeta
}

\section{Together We Shape the Future of the Planet}

Sergio Fernández Valdés ${ }^{2}$ N Nicolás Piñeros Barrera ${ }^{3}$

\section{Resumen}

El artículo reflexiona y propone cómo se podría generar conciencia sobre el estado actual del medioambiente y la importancia de cuidarlo. Se analizó una investigación realizada por la Universidad del Norte, donde observaron que los estudiantes de primaria decían llevar a cabo prácticas de reciclaje. Sin embargo, la gran mayoría no lo hacían en realidad. Se realizó una encuesta a niños y niñas de 9 a 15 años de edad, en donde se evidenció que los menores conocen las formas en las cuales se destruye el medioambiente y también saben cómo deben cuidarlo. También se desmostró que algunos afirman reciclar junto a sus padres, lo que demuestra una cultura de cuidado del medioambiente, y se concluyó que es posible generar conciencia para que, tanto padres como hijos, actúen en conjunto para cuidar el medioambiente desde sus hogares, forjando un futuro mejor para todos.

Palabras clave: medio ambiente, contaminación, conciencia, problemas.

\section{Abstract}

The article reflects on and proposes how to raise awareness of the current environmental condition and the importance of protecting the environment. We analyzed a study carried out by the Universidad del Norte, in which elementary school students claimed to follow recycling practices, but the vast majority actually did not. A survey was conducted with children aged 9 to 15 , finding that they know how we damage the environment and how to care for it. We noted that some say they recycle with their parents, thus demonstrating a culture of caring for the environment. In conclusion, it is possible to raise awareness by involving both parents and

2 Estudiante Profesional de Entrenamiento Deportivo, Fundación Universitaria del Área Andina.

Correo: sfernandez14destudiantes.areandina.edu.co

3 Estudiante Entrenamiento Psicología, Fundación Universitaria del Área Andina. Correo: npinerosb五estudiantes.areandina.edu.co 
children in taking care of the environment from their homes, thus shaping a better future for all.

Keywords: Environment, Pollution, Awareness, Problems.

\section{Introducción}

La crisis ambiental que existe hoy en día está generando una gran controversia en la gente del común. Por otro lado, hay muchas personas que sin importar los problemas ecológicos de la actualidad siguen llevando un estilo de vida egoísta y desinteresado, generando una mayor contaminación lo que demuestra una ausencia de conciencia social. Para poder lograr un progreso y una mayor conciencia sobre esta problemática deben tenerse en cuenta los siguientes cuatro aspectos (Martínez, 2008):

- Cognitiva: dentro de esta se evalúa o interpreta el grado de conocimientos sobre el medioambiente que posee la persona o grupo de personas.

- Afectiva: cómo la persona se relaciona con el medio ambiente, cómo percibe este y de igual forma cómo se relaciona con el mismo, lo cual dará cierta claridad sobre qué tan relacionada está la persona con su medio.

- Conativa: la disposición de la persona en cuestión sobre cuánto quiera involucrarse en temas proambienta- les o relacionados con el cuidado de la naturaleza.

- Activa: se refiere al comportamiento de la persona, qué actividades realiza esta para el cuidado del medio ambiente, así como aquellas conductas que hace por el beneficio del entorno.

Debe tenerse en cuenta que la cultura se forja desde pequeños, por ende, debemos enfocarnos en generar una mayor conciencia en los niños y adolescentes, para que estos en un futuro fomenten el desarrollo de la misma conciencia que adquirieron en su infancia. Más cuando el medio ambiente emerge como un espacio de intervención subjetiva, como un elemento de constitución social y como ámbito normativo que propicia e inhibe la valoración, la percepción, la conciencia, la protesta y la reivindicación social (Lezama, 2004).

\section{Marco teórico}

\section{Análisis de antecedentes}

Hay que tener en cuenta que la idea de generar conciencia en los niños y adolescentes no es algo nuevo, ya se han vis- 
to proyectos similares en donde se buscan dos cosas: conocer qué tanto saben los niños respecto al medio ambiente y sus cuidados, así como incentivar a que aprendan las diversas formas de cuidarlo y que este comportamiento y aprendizaje no se limite únicamente a su vida escolar sino también lo apliquen en otros ámbitos de su vida. Un ejemplo de esto es un artículo de investigación de la Universidad del Norte, en donde el objetivo era dar a conocer qué tanto sabían los niños y niñas de colegios públicos de la ciudad de Santa Marta. En dicho estudio se encontró que un $77 \%$ de los niños y niñas de quinto de primaria que fueron entrevistados decían clasificar los residuos para hacer un apropiado reciclaje, pero cuando se les observó en el entorno escolar se descubrió que esto no era del todo cierto y se llegó a la conclusión que la participación de estos en comportamientos de protección del medio ambiente es realmente baja (Cabezas y Barrios, 2014).

Teniendo en cuenta la anterior investigación, hay que identificar la principal problemática, que es que aunque los niños en la mayoría de los casos pueden aprender cómo cuidar el medio ambiente y las formas para que esto haga la diferencia, muchas veces no tienen alguna motivación adicional que los impulse a realizar estos cambios o en ocasiones los propios padres no apoyan lo suficiente esta clase de conductas en el joven, haciendo que estos muchas veces pierdan el impulso que tenían. Después de identificar uno de los obstáculos, ahora tenemos que evidenciar los problemas ambientales presentes en Colombia, así como qué tan desinformados están los niños y niñas del país.

Antes de hablar sobre cómo vamos a llevar a cabo los procesos de concientización y motivación en los niños y niñas tenemos que conocer los problemas medioambientales que afectan a todos los colombianos por igual. Teniendo en cuenta lo anterior, podemos decir — gracias a estudios realizados por diversas universidades del país - que Colombia presenta las siguientes afectaciones medioambientales (Ruiz, 2018, Gómez, 2018, Quintero y Jiménez, 2017 y Martínez, 2017).

- Contaminación de aire principalmente en Bogotá y Medellín.

- Contaminación de fuentes hídricas en Casanare, Quindío y municipios aledaños.

- Contaminación visual en Bogotá, específicamente en sector de Quiriguá.

Ya habiendo identificado algunos de los principales problemas medioambientales que tenemos en Colombia, debe- 
mos hablar de cómo se puede lograr que los niños más pequeños adopten conductas de cuidado con su entorno. Para empezar, debe tenerse en cuenta que los seres humanos vivimos y actuamos únicamente por motivación, en pocas palabras si algo no nos da curiosidad o no nos da ningún tipo de motivación para hacerlo, simplemente no lo hacemos. Teniendo en cuenta esto y un estudio realizado por la Universidad Técnica de Ambato (el cual se centró en cómo estimular la educación del reciclaje en niños y niñas de sexto grado) podemos decir que no es tarea fácil hacer que un niño o adolescente sienta alguna atracción o motivación hacia el cuidado del medio ambiente, para la cual se deben obtener datos sobre (Nasimba, 2015):

1. ¿Qué tanto conocen sobre el medio ambiente?

2. ¿Qué formas de proteger el ambiente conocen?

3. ¿Cómo harían ellos para cuidar el medio ambiente desde sus hogares?

Esta clase de preguntas serían las apropiadas para idear formas adecuadas de acercarnos a los infantes y poder lograr en ellos que su aprendizaje y sus experiencias resulten significativas. De este modo, pueden dejar huella desde pequeños, tanto en sus hogares de for- ma individual como en sus comunidades de forma colectiva y social, con el fin de que puedan tener un mejor desarrollo, un ambiente más limpio y un mejor futuro para todos. Se podrían hacer diversas actividades, tales como, obras de teatro, shows con títeres, canciones educativas, actividades lúdicas, entre otras actividades que dejen una huella en sus mentes.

\section{Resultados}

La encuesta fue diligenciada por 22 niños y niñas entre los 9 y 15 años de edad, siendo 14 años el mayor grupo de niños que respondieron la encuesta. De los 22 encuestados, 15 de ellos viven en Bogotá D.C. y 7 en el municipio de Funza. En la encuesta se preguntó sobre el nivel académico más alto o por el cual están cursando actualmente, de esto se obtuvo que una gran parte de nuestros encuestados están actualmente en grado noveno, mientras que los demás están actualmente en quinto, sexto, séptimo, entre otros.

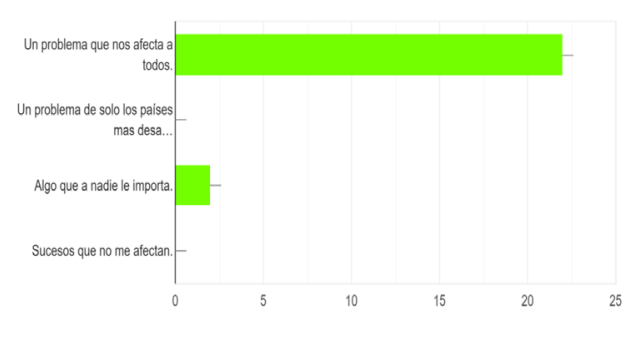

Figura 1. ¿Qué es un problema ambiental? Fuente: elaboración propia. 
Los 22 niños y niñas dando un total del $100 \%$, dijeron que es un problema que nos afecta a todos. Dos dicen que es algo que a nadie le importa con un 9,1 \%. Mediante estos resultados podemos observar que los infantes tienen presente que un problema medioambiental es un problema de todos. Esto es algo bueno, porque demuestra que por lo menos poseen algo de información sobre el tema.

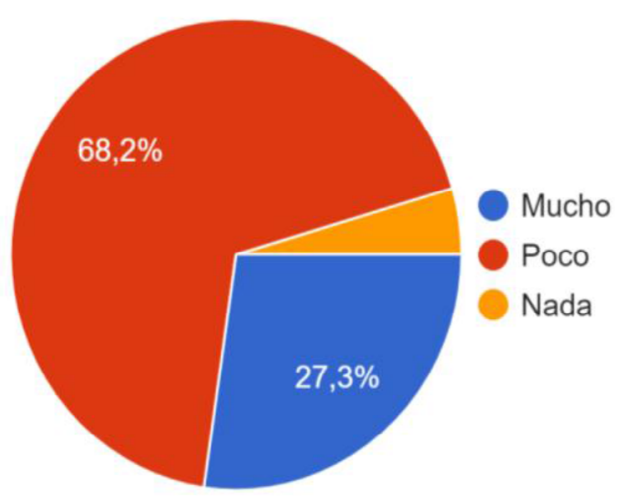

Figura 2. ¿Cuánto sabes sobre el medio ambiente?

Fuente: elaboración propia.

Se les preguntó cuánto saben del medio ambiente, de los 22 niños y niñas 15 dijeron que sabían poco con un 68,2 $\%$; 6 consideraron que saben mucho con un $27,3 \%$ y solo 1 , que nada con el 4,5 $\%$. Es verdaderamente preocupante observar que con honestidad la gran mayoría saben tan poco al respecto.

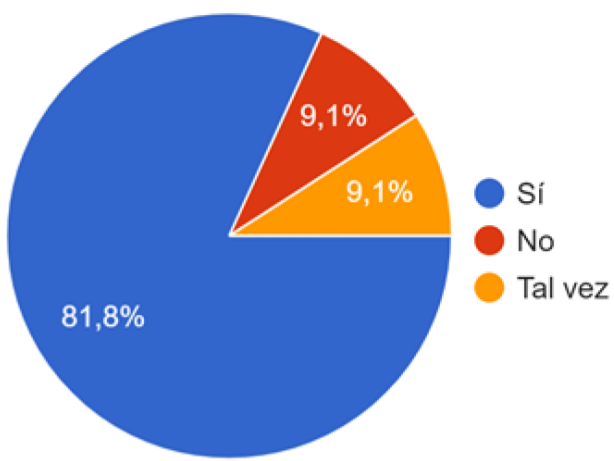

Figura 3. ¿Te interesaría conocer sobre el medioambiente?

Fuente: elaboración propia.

En la figura 3 pueden observarse los resultados sobre si les interesaría conocer sobre el medio ambiente. De los 22 participantes, 18 de ellos dijeron que sí con un gratificante $81,8 \%$ y 2 de ellos respondieron no con un 9,1\%. Por último, 2 respondieron tal vez con un 9,1 $\%$. Es realmente importante saber que la gran mayoría de niños presentan un interés y les gustaría aprender más sobre el medio ambiente.

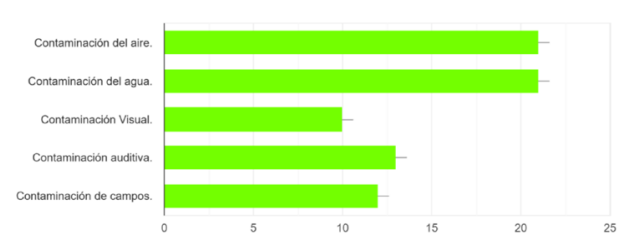

Figura 4. ¿Cuáles crees que son los problemas ambientales de Colombia? Fuente: elaboración propia. 
A los 22 participantes se les preguntó cuáles son los problemas ambientales en Colombia. Les dimos varias opciones, donde podrían marcar más de una: A) Contaminación del aire con un total de 21 participantes y un 95,5\%. B) Contaminación del agua con un total también de 21 participantes y un 95,5\%. C) Contaminación visual con unos 10 participantes con un 45,5\%. D) Contaminación auditiva con 13 participantes con $59,1 \%$. E) Contaminación de campos con 12 participantes con un 54,5\%.

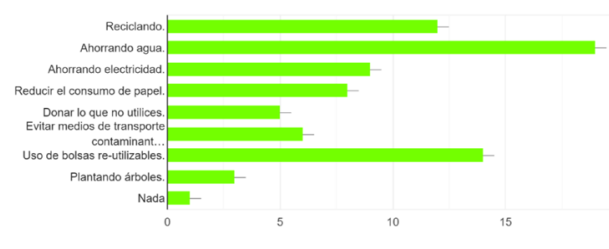

Figura 5. ¿De qué formas cuidas el medio ambiente?

Fuente: elaboración propia.

A los 22 participantes se les dio varias opciones para que marcaran de qué forma cuidan el medio ambiente. A) Reciclar,12 de ellos con un 54,5\%. B) Ahorrar agua, 19 de ellos con un 86,4 \%. C) Ahorrar electricidad, 9 de ellos y un 40,9\%. D) Reducir el consumo de papel, 8 de ellos y con $36,4 \%$. E) Donar lo que no utilices, 5 de ellos y un 22,7\%. F) Evitar medios de transporte contaminantes, 6 de ellos con un $27,3 \%$. G) Uso de bolsas reutilizables, 14 de ellos con un 63,6\%. H) Plantar árboles, 3 de ellos con un $13,6 \%$. I) Nada, 1 solo fue el que eligió nada con un 4,5\%.

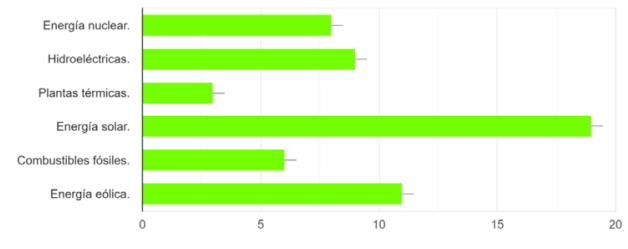

Figura 6. ¿Qué formas de energía renovable conoces?

Fuente: elaboración propia.

A los encuestados se les preguntó qué formas de energía renovables conocen, con respuestas múltiples: A) Energía nuclear, 8 de ellos con un 36,4\%. B) Hidroeléctricas, 9 de ellos con 40,9\%. C) Plantas térmicas, 3 de ellos con 13,6\%. D) Energía solar, 19 de ellos con un 86,4\%. E) Combustibles fósiles, 6 de ellos con un 27,3\%. F) Energía eólica, 11 de ellos con $50 \%$.

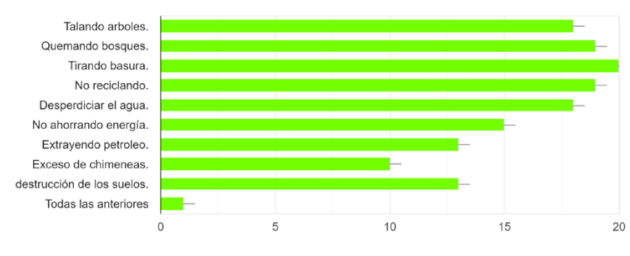

Figura 7. ¿De qué forma NO cuidamos el medio ambiente?

Fuente: elaboración propia.

A los 22 participantes se les preguntó de qué formas no cuidaban el medio am- 
biente y le dimos varias respuestas con selección múltiples. A) Talar árboles, 18 de ellos con un 81,8\%. B) Quemar árboles, 19 de ellos con 86,4\%. C) Botar basura, 20 de ellos con un 90,9\%. D) No reciclar, 19 de ellos con un $86,4 \%$. E) Desperdiciar el agua,18 de ellos con un $81,8 \%$. F) No ahorrando energía,15 de ellos con un $68,2 \%$. G) Extrayendo petróleo,13 de ellos con un 59,1\%. H) Exceso de chimeneas, 10 de ellos con un 45,5\%. I) Destrucción de los suelos, 13 de ellos con un 59,1\%. J) Todas las anteriores, 1 de ellos con 4,5\%.

¿Cómo cuidas el medio ambiente desde tu hogar?

Mediante los resultados pudimos evidenciar que en la mayoría de las familias separan los residuos reciclables de los que no lo son. También pudimos observar que se hace un ahorro de agua y luz, así como que algunos reutilizan bolsas plásticas para así generar un menor impacto ambiental. Con esto podemos ver que en los hogares colombianos se realizan algunos aportes al cuidado del medioambiente. Esto es algo verdaderamente positivo, sin embargo, puede mejorar.

¿Qué consecuencia tiene la contaminación en todas sus formas?

A los encuestados se les preguntó qué consecuencia tiene la contamina- ción en todas sus formas y respondieron, con mucha franqueza, que podría ser el fin de la tierra. Algunos concordaron en que la contaminación haría que el oxígeno en un futuro sea poco respirable. De igual forma mencionaron las graves consecuencias a la salud humana que tiene la contaminación. También opinaron que de tanta contaminación a nivel mundial, la capa de ozono de la tierra puede llegar a dañarse hasta desaparecer generando un daño completamente irreparable.

¿Qué crees tú que es el cambio climático?

A los niños y jóvenes se les preguntó si sabían qué es el cambio climático. Respondieron abiertamente. La mayoría dieron respuestas similares a que el cambio climático es generado por elementos contaminantes que afectan a la tierra, el agua y el aire haciendo que el clima cambie. Puede definirse el cambio climático como la variación en el estado del sistema climático, formado por la atmósfera, la criosfera, la hidrosfera, la biosfera y litosfera.

¿Qué tanto saben tus padres sobre el cambio climático?

Una de las últimas preguntas era: ¿Qué tanto saben sus padres respecto al cambio climático? Dependiendo a las respuestas que dieron, evidenciamos 
que la mayoría de los padres no tienen muchos conocimientos respecto al tema. Sin embargo, algunos pocos de los encuestados afirman que sus padres saben bastante sobre el cambio climático y sus consecuencias. Lo poco que saben es porque ven el noticiero $u$ otros medios de comunicación; eso da a entender que es muy importante generar charlas o espacios donde se brinde información a todos los públicos para que se genere conciencia e interés.

\section{¿Estamos en problemas y por qué?}

En la última pregunta de la encuesta se pidió la opinión de cada uno de los participantes, la mayoría respondió que estamos en un verdadero problema debido a la poca conciencia que existe al respecto y al poco interés que le prestamos a esta clase de cosas, sin importar que el cambio climático sea un problema que nos afecta a todos por igual. También estamos en problemas porque el nivel del mar está subiendo y los casquetes polares se están fundiendo ya que en algunas regiones se registran olas de calor, sequía y las inundaciones son cada vez más frecuente.

\section{Conclusiones}

Podemos concluir que algunos niños y niñas de Colombia saben, o al menos conocen, aspectos generales sobre cuáles son los factores que ayudan al cuidado del medio ambiente y cuáles son aquellos que generan su destrucción. Esto es verdaderamente alentador porque se puede ver que las nuevas generaciones no pasan por alto los temas relacionados con el ambiente y reconocen que este debe ser preservado.

También se puede concluir que existe una cultura de reciclaje donde los padres fomentan en el niño el separar los residuos. De esta forma se está generando una conciencia de cambio; pensamientos e ideas que irán pasando de padres a hijos para generar una cultura de cambio enfocada al cuidado del medio ambiente.

De igual forma es importante denotar cómo los más pequeños del hogar perciben su entorno, el mundo que los rodea y cómo día tras día que avanza la civilización, el ecosistema se afecta más por la mano humana tras la búsqueda del recurso energético, sin importar el daño que le puedan generar al planeta con plantas de energía nuclear, por ejemplo, las cuales brindan un gran recurso energético a costa de contaminar la tierra y el agua.

En conclusión, los menores pueden cambiar el rumbo de la civilización, ya 
que la gran mayoría de los encuestados no solo presentan un interés sobre el medio ambiente, sino también les importa saber cómo preservarlo. De esta forma, poco a poco, llegando a cada una de sus pequeñas mentes, generando la conciencia y el impacto adecuado, se puede cuidar la vida en la tierra. No solo para preservar la existencia humana, sino también para poder vivir en armonía con el planeta y con nosotros mismos, y lograr, de esta forma, forjar juntos el futuro del planeta.

\section{Referencias}

Cabezas, M. R. R., y Barrios, E. S. P. (2014). Educación ambiental en niños y niñas de instituciones educativas oficiales del distrito de Santa Marta. Zona Próxima, (21), 52-64, de https://www.redalyc.org/ pdf/853/85332835005.pdf

Gómez, C. A. (2018). Contaminación del aire de Medellín por pm10 y pm2.5 y sus efectos en la salud, de https://repository.unimilitar.edu.co/handle/10654/17019

Lezama, J. L. (2004). La construcción social y política del medio ambiente. El Colegio de México AC, de https:// books.google.es/books?hl=es\&lr=\&id=$\mathrm{J} 1 \mathrm{o} \mathrm{jRh} 751 \mathrm{EC} \& \mathrm{oi}=$ fnd\&pg $=$ PA98\&d$\mathrm{q}=$ Lezama, $+\mathrm{J} .+\mathrm{L} .+(2004) .+\mathrm{La}+$ cons trucci\%C3\%B3n+social $+\mathrm{y}+$ pol $\% \mathrm{C} 3 \% \mathrm{~A}-$ Dtica+del+medio+ambiente. + El+Colegio + de + Mexico + AC.\&ots=BMIqvDQmFr\&sig=3ygv8ixjsmK8zHveOz5uuD-

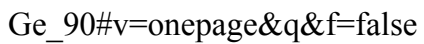

Martínez, A. G. (2008). La conciencia ambiental como herramienta para la educación ambiental: conclusiones y reflexiones de un estudio en el ámbito universitario. Universidad de Córdoba, de https://www. miteco.gob.es/en/ceneam/articulos-de-opinion/2008_11gomera1_tcm38-163624.pdf

Martínez, S. J. (2017). Factores de contaminación ambiental por el desarrollo de proyectos petroleros en el Caño el Ingeniero, municipio de Puerto Gaitán, Meta, de http:// ridum.umanizales.edu.co:8080/xmlui/ handle/6789/3143

Nasimba, M. G. (2015). La estimulación educativa sobre el reciclaje y su influencia en la protección del medio ambiente de los niños y niñas de los sextos grados de Educación Básica de la Escuela Fiscal Mixta Isidro Ayora de la Parroquia de Uyumbicho, Cantón Mejía, Provincia de Pichincha (Bachelor's thesis, Universidad Técnica de Ambato. Facultad de Ciencias Humanas y de la Educación. Carrera de Educación Básica), de https://repositorio.uta.edu.ec/ handle/123456789/13298

Quintero, S. y Jiménez, N. (2017). Diagnóstico de los elementos atípicos asociados a la contaminación visual presentes en la zona urbana de Funza y los identificados en el sector Quiriguá de Bogotá, de https:// ciencia.lasalle.edu.co/ing_ambiental_sanitaria/482/

Reyes, Y., Vergara, I., Torres, O., Lagos, M. D., \& Jiménez, E. E. G. (2016). Contaminación por metales pesados: implicaciones en salud, ambiente y seguridad alimentaria. Ingeniería Investigación y Desarrollo: $\mathrm{I} 2+$ D, 16(2), 66-77, de https://dialnet.unirioja. es/servlet/articulo?codigo $=6096110$ 
Ruiz, D. A. (2018). Vulnerabilidad a la contaminación de los acuíferos someros con porosidad primaria, departamento del Quindío, Colombia, de https://expeditiorepositorio. utadeo.edu.co/handle/20.500.12010/4463 